\title{
Patients with End-Stage Renal Disease are at Increased Risk of Postoperative Complications Following Lower Extremity Fracture Surgery
}

Atsushi Endo MD, Michael F. McTague MPH, Elizabeth M. Allen BS, Arvind von Keudell MD, Michael J. Weaver MD

Investigation Performed at: BWH and MGH

\section{Introduction}

End-stage renal disease (ESRD) leads to multiple systemic effects and patients suffer from multiple comorbidities including fractures. While previous studies have examined complications following hip fracture surgery in ESRD patients, there are no studies evaluating other lower extremity injuries. This study aimed to identify postoperative complication risk in patients with ESRD who had lower extremity fractures.

\section{Methods}

We performed a retrospective matched cohort study. Using our database from 2000 to 2015 at two level-one trauma centres, we collected data on patients over age 40, who had lower extremity fractures and surgical fixation. Diagnosis of ESRD was made before the injury. Each ESRD patient was matched by two non-ESRD patients regarding age, gender, American Society of Anaesthesiologists (ASA) score, and AO/OTA fracture classification.

Postoperative outcomes were non-union (as defined by the treating surgeon), mechanical failure (radiographic loss of reduction or implant failure requiring revision surgery), and infection (based upon clinical evaluation and requiring either admission for IV antibiotics or surgical treatment). The number of outcome events was compared between the ESRD and non-ESRD cohorts.

\section{Results}

A total of 195 patients (65 ESRD patients matched to 130 non-ESRD patients) were identified. Mean age was 69 years old (range 42 to 99 years old). Median follow-up was 31 months (range 12 to 141 months).

Patients with ESRD were significantly more likely to have at least one postoperative complications (mechanical failure, non-union, and infection) compared to non-ESRD patients (9/65 vs. $5 / 130, p=0.02)$. Mechanical failure was significantly more common among ESRD patients compared to nonESRD patients (8/65 vs. $2 / 130, p<0.01)$. While there was a slightly higher risk of nonunion and infection in the ESRD patients this was not statistically significant.

\section{Conclusion}

ESRD was associated with higher rates of complications, especially mechanical failure, after lower extremity fracture surgeries.

\section{Disclosures}

No funding was received in support of this project. The Authors have no financial disclosures.

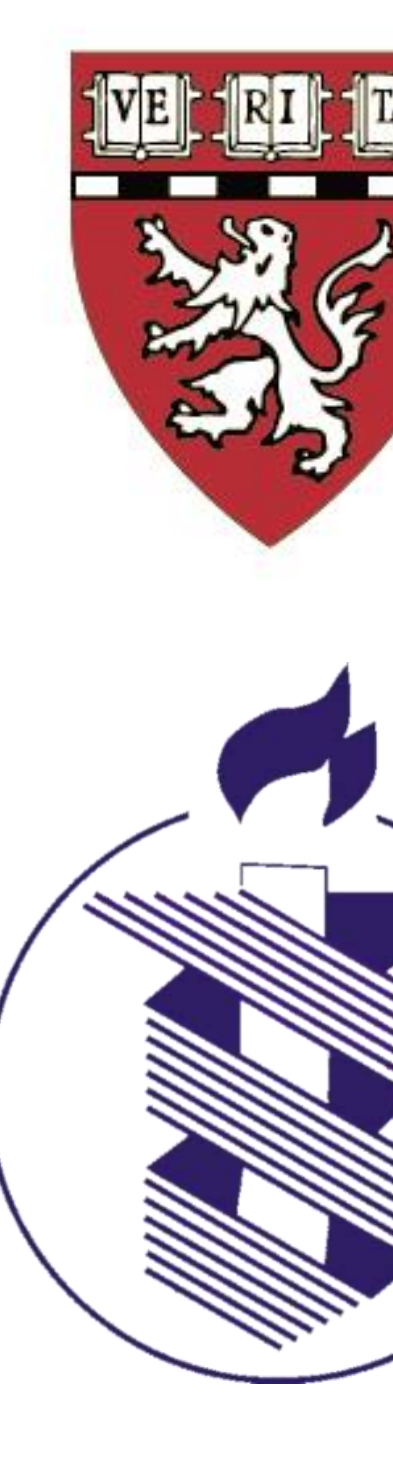

ORTHOPEDIC TRAUMA INITIATIVE HARVARD MEDICAL SCHOOL

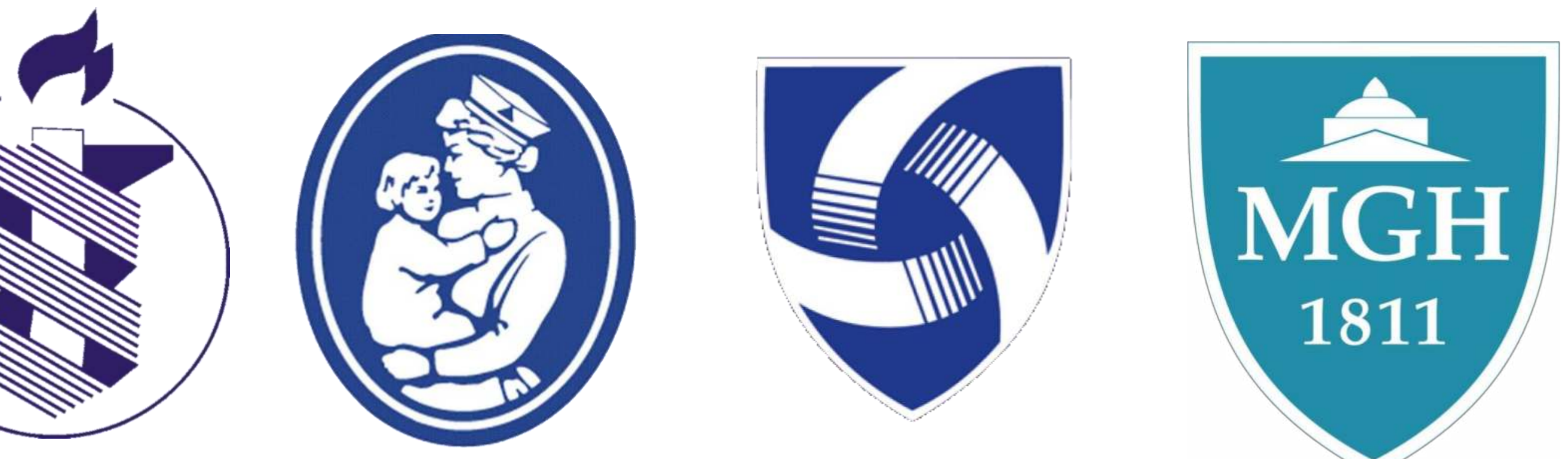

Patient Characteristics

\begin{tabular}{|c|c|c|}
\hline & $\begin{array}{l}\text { ESRD } \\
(N=65)\end{array}$ & $\begin{array}{l}\text { Non-ESRD } \\
(\mathrm{N}=130)\end{array}$ \\
\hline Age - year (SD) & $69+/-12$ & $69+/-12$ \\
\hline Female sex - no. (\%) & $40(62)$ & $80(62)$ \\
\hline \multicolumn{3}{|l|}{ ASA score - no. (\%) } \\
\hline 2 - mild systemic disease & $4(6)$ & $8(6)$ \\
\hline 3 - severe systemic disease & $55(85)$ & $110(85)$ \\
\hline 4 - constant threat to life & $6(9)$ & $12(9)$ \\
\hline \multicolumn{3}{|l|}{ AO/OTA classification - no. (\%) } \\
\hline 31 - proximal femur & $42(64)$ & $84(64)$ \\
\hline 32 - diaphyseal femur & $3(5)$ & $6(5)$ \\
\hline 33 - distal femur & $3(5)$ & $6(5)$ \\
\hline 41 - proximal tibia/fibula & $2(3)$ & $4(3)$ \\
\hline 42 - diaphyseal tibia/fibula & $0(0)$ & $0(0)$ \\
\hline 43 - distal tibia/fibula & $3(5)$ & $6(5)$ \\
\hline 44 - malleolar & $12(18)$ & $24(18)$ \\
\hline Smoking - no. (\%) & $11(17)$ & $23(18)$ \\
\hline Steroid use - no. (\%) & $10(15)$ & $7(5)$ \\
\hline \multicolumn{3}{|l|}{ Charlson index - no. (\%) } \\
\hline $0-1$ & $0(0)$ & $61(47)$ \\
\hline $2-3$ & 9 (14) & $45(35)$ \\
\hline 4-5 & $41(63)$ & 20 (15) \\
\hline 6-7 & $15(23)$ & 4 (3) \\
\hline $\begin{array}{l}\text { Follow-up months (median, } \\
\text { Interquartile Range) }\end{array}$ & $39(21-73)$ & $30(22-48)$ \\
\hline
\end{tabular}

Postoperative Complications

\begin{tabular}{|l|c|c|c|c|} 
& $\begin{array}{c}\text { ESRD } \\
(\mathrm{N}=65)\end{array}$ & $\begin{array}{c}\text { Non-ESRD } \\
(\mathrm{N}=130)\end{array}$ & RR & P Value \\
\hline Non-union - no. (\%) & $3(5)$ & $2(2)$ & 3 & 0.34 \\
\hline Mechanical failure - no. (\%) & $8(12)$ & $2(2)$ & 8 & 0.003 \\
\hline Post-op infection - no. (\%) & $4(6)$ & $3(2)$ & 2.7 & 0.22 \\
\hline Composite - no. (\%) & $9(14)$ & $5(4)$ & 3.6 & 0.02 \\
\hline
\end{tabular}

References

1. Karaeminogullari O, Demirors $\mathrm{H}$, Sahin $\mathrm{O}$, et al. Analysis of outcomes for surgically treated hip fractures in patients undergoing chronic hemodialysis. J Bone Joint Surg Am. 2007;89(2):324-31.

2. Lin JC, Liang WM. Mortality and complications after hip fracture among elderly patients undergoing hemodialysis. BMC nephrol. 2015;16:100.

3. Tosun B, Atmaca H, Gok U. Operative treatment of hip fractures in patients receiving hemodialysis. Musculoskelet surg. 2010;94(2):71-5. 